\title{
Neue Daten aus Phase-III-Studien zu Ixekizumab
}

Beim diesjährigen Kongress der American Academy of Dermatology (AAD) Anfang März in Washington wurden Daten aus den Phase-III-Studien UNCOVER-2(1) und UNCOVER-3(1,2) vorgestellt. Ergebnis: Menschen mit mittelschwerer bis schwerer Plaque-Psoriasis können unter Behandlung mit dem Interleukin(IL)-17A-Inhibitor Ixekizumab ein hohes Maß an Erscheinungsfreiheit erreichen.

Ixekizumab ist ein monoklonaler Antikörper, der als subkutane Injektion (80 mg) mit einem Fertigpen oder einer Fertigspritze verabreicht wird und mit hoher Affinität und Spezifität an IL-17A bindet und dieses neutralisiert.

Am 25. April hat Ixekizumab (Handelsname Taltz ${ }^{\circ}$ ) die Zulassung in Europa erhalten. Indiziert ist der Interleukin(IL)-17A-Inhibitor zur Behandlung von Erwachsenen mit mittelschwerer bis schwerer Plaque-Psoriasis, die für eine systemische Therapie in Frage kommen.

\section{Die aktuellen Studien}

UNCOVER-2 und UNCOVER-3 sind doppelblinde, multizentrische Phase-III-Studien, an denen seit 2012 über 2500 Patienten teilnehmen. Die Patienten erhielten über einen Zeitraum von 12 Wochen entweder Placebo, Etanercept (50 mg zweimal wöchentlich) oder Ixekizumab (80 mg alle zwei bzw. vier Wochen, in beiden Gruppen nach einer Anfangsdosis mit 160 mg).

\section{Verbesserung des Hautbilds unter Ixekizumab}

Patienten können unter Ixekizumab ein hohes Maß an Erscheinungsfreiheit erreichen. Das zeigen die Ergebnisse von UNCOVER-2 [2] und -3 [3] aus den ersten 12 Wochen. Etwa $90 \%$ der Patienten, die zweiwöchentlich mit Ixekizumab $(80 \mathrm{mg}$ ) behandelt wurden, erreichten einen PASI 75 , etwa $70 \%$ eine nahezu erscheinungsfreie Haut
(PASI 90) und rund $40 \%$ der Patienten eine völlig erscheinungsfreie Haut (PASI 100). Die beim AAD-Kongress vorgestellten Subanalysen untermauern die gute Wirksamkeit von Ixekizumab.

Detaillierte Auswertungen der UNCOVER-2-Studie [1] betrachteten diejenigen Patienten aus der Vergleichsgruppe, die innerhalb von 12 Wochen nicht auf die Behandlung mit Etanercept (50 mg zweimal wöchentlich) ansprachen. Insgesamt entsprach dies $64 \%$ der Patienten [4]. In Woche 12 erhielten diese Patienten Placebo. Danach wurden sie von Woche 16 bis Woche 60 alle vier Wochen mit Ixekizumab $(80 \mathrm{mg})$ behandelt. Unter Ixekizumab erreichten sie eine deutliche Verbesserung des Hautbilds: Nach 12 Wochen Behandlung mit Ixekizumab (28 Wochen Studiendauer) erreichten $83,5 \%$ der Patienten einen PASI 75, $57 \%$ einen PASI 90 und $22,0 \%$ einen PASI 100. Nach 44 Wochen Behandlung mit Ixekizumab (60 Wochen Studiendauer) erreichten $82,5 \%$ der Patienten einen PASI 75, 68,5\% einen PASI 90 sowie 43,5\% einen PASI 100.

„Zu Ixekizumab wurden in Washington fast 30 Poster vorgestellt. Die guten Daten zur Erscheinungsfreiheit sowie Geschwindigkeit, mit der Behandlungsergebnisse sichtbar werden, lassen auf ein großes Potenzial des IL-17A-Inhibitors hoffen," fasste Reich zusammen.

\section{Sichtbare Ergebnisse nach einer Woche}

Dass Ixekizumab schnell wirkt und nach einer Woche zu sichtbaren Effekten führen kann, belegen Daten aus UNCOVER-2 und -3 [2]. In einer kombinierten Analyse wurden die Zeit bis zum Eintreten einer klinischen Verbesserung (gemessen anhand der mittleren prozentualen Verbesserung des PASI vom Ausgangswert), die Zeit bis zum Erreichen von PASI 50 sowie von PASI 75 im Vergleich zum Ausgangswert unter Ixekizumab (alle zwei Wochen), Etanercept und Placebo gemessen. Die Daten zeigen signifikante Unterschiede zwischen den Gruppen.

Bereits nach einer Woche erreichten unter Ixekizumab deutlich mehr Patienten eine signifikante Verbesserung des Hautbilds um $50 \%$ im Vergleich zu Etanercept und Placebo. Die durchschnittliche Zeit bis zum Erreichen eines PASI 75 lag unter Ixekizumab bei 30 Tagen, unter Etanercept waren es 85 Tage.

\footnotetext{
Literatur

1. Papp K et al (2016) Poster 3017 AAD, Washington, 04.-08. März 2016.

2. Leonardi C et al (2016) Poster 3100 AAD, Washington, 04.-08. März 2016.

3. Griffiths CEM et al (2015) Lancet 386(9993):541-551

4. Lilly data on file
}

hautnah $2016 \cdot 15: 25$

DOI 10.1007/s12326-016-0194-4

Online publiziert: 2. Mai 2016

() Springer-Verlag Wien 2016

Quelle:

Pressemitteilung

Eli Lilly 